\title{
Conodont alteration and tectonothermal evolution of a diagenetic unit in the Iberian Variscan belt (Ponga-Cuera unit, NW Spain)
}

\author{
SILVIA BLANCO-FERRERA*†, JAVIER SANZ-LÓPEZ , SUSANA GARCÍA-LÓPEZ*, \\ FERNANDO BASTIDA* \& M. LUZ VALÍN* \\ *Departamento de Geología, Universidad de Oviedo, 33005 Oviedo, Spain \\ $\ddagger$ Facultade de Ciencias da Educación, Universidade da Coruña, 15071 A Coruña, Spain
}

(Received 26 July 2009; accepted 3 February 2010; first published online 3 June 2010)

\begin{abstract}
Tectonothermal analysis of a mainly carbonate unit located in the external part of the Variscan orogen in NW Spain is dealt with using the conodont colour alteration index (CAI) and the study of textures of these microfossils. The Kübler index of the illite (KI) is used as a complementary method. The area is characterized by a great uniformity in the CAI values, which in most cases are $<2$, indicating diagenetic conditions. In spite of the low CAI values, textures show great variety and were mainly originated by diagenetic processes of apatite dissolution and precipitation. The conodonts underwent a long period of heating (probably from the Pennsylvanian to the Cenozoic) to low temperatures $\left(<100^{\circ} \mathrm{C}\right)$ to reach the low CAI values measured. Assuming a normal geothermal gradient, these temperatures required an overburden $<3 \mathrm{~km}$ that in part was due to burial and in part to tectonic superimposition. Minor local anomalies in the CAI values and some textural alterations, related to dissolution and precipitation of authigenic minerals, could be due to epithermal activity that gave rise to various ore deposits in the studied area mainly during Permian times.
\end{abstract}

Keywords: diagenesis, CAI, conodont textures, clay minerals, Variscan.

\section{Introduction}

Two important factors in the processes that give rise to the development of orogenic belts are temperature and the presence of fluids. Mapping of index values of thermal indicators in diagenetic areas, together with analysis of features that show fluid activity, may facilitate an understanding of their tectonothermal evolution, and the evaluation of potential hydrocarbon resources and ore deposits. Several methods may be used for this purpose, such as the conodont colour alteration index (CAI), microscopic analysis of the textural modifications of these microfossils, the illite Kübler index (KI), X-ray diffraction analyses of clay mineralogy, vitrinite reflectance $(\mathrm{Rr} \%)$, coal rank, palynomorph colour indices and the study of fluid inclusions.

Within this line of research, the aim of the present study is to establish the distribution of the maximum palaeotemperatures, based on CAI data, and to detect the fluid activity, mainly using the textures of conodonts, in a diagenetic area (the Ponga-Cuera unit) located in NE part of the Cantabrian zone (Fig. 1), which represents the Variscan foreland fold-and-thrust belt in NW Spain. The results are compared with those obtained with other thermal indicators and related to the lithology, structure and ore deposits of the area. Finally, an evolutionary model of the tectonothermal processes that occurred in the area is presented.

The area mainly consists of limestone and quartz arenite, hence the main indicator used is the CAI.

$\nmid$ †uthor for correspondence: silvia.blanco@geol.uniovi.es
None the less, CAI data have been complemented by Kübler index data (KI) and clay mineralogy studies of samples collected in the less common shale outcrops present in the area. Results obtained by other authors for coal rank and vitrinite reflectance (Colmenero et al. 2008) in a Carboniferous unconformable siliciclastic band located outside of the studied area, although close to its southern boundary, are also taken into account. The interpretations made from the results obtained are placed in the context of the geology of the region and studies related to the tectonothermic evolution of the Cantabrian zone, particularly those interpretations concerning the adjacent unit of the Picos de Europa (Bastida et al. 2004).

\section{Geological setting}

The Ponga-Cuera unit has an E-W trend, and is bounded on the north by the Cantabrian coast (Figs 1, 2). To the south it thrusts over the Picos de Europa unit, mainly formed by Carboniferous limestones affected by a south-facing imbricate thrust system. In the southern part of the Picos de Europa unit, a palaeothermal gradient increasing southwards has been found, such that the boundary between the diacaizone and ancaizone (palaeothermal zones defined by CAI values after García-López et al. 2001) appears near their basal thrust (Bastida et al. 2004). To the west, the rocks of the Central Coal basin thrust over the Ponga-Cuera unit, which is a unit mainly formed by a thick siliciclastic Carboniferous succession. To the east, the study area is bounded by the rocks of the 


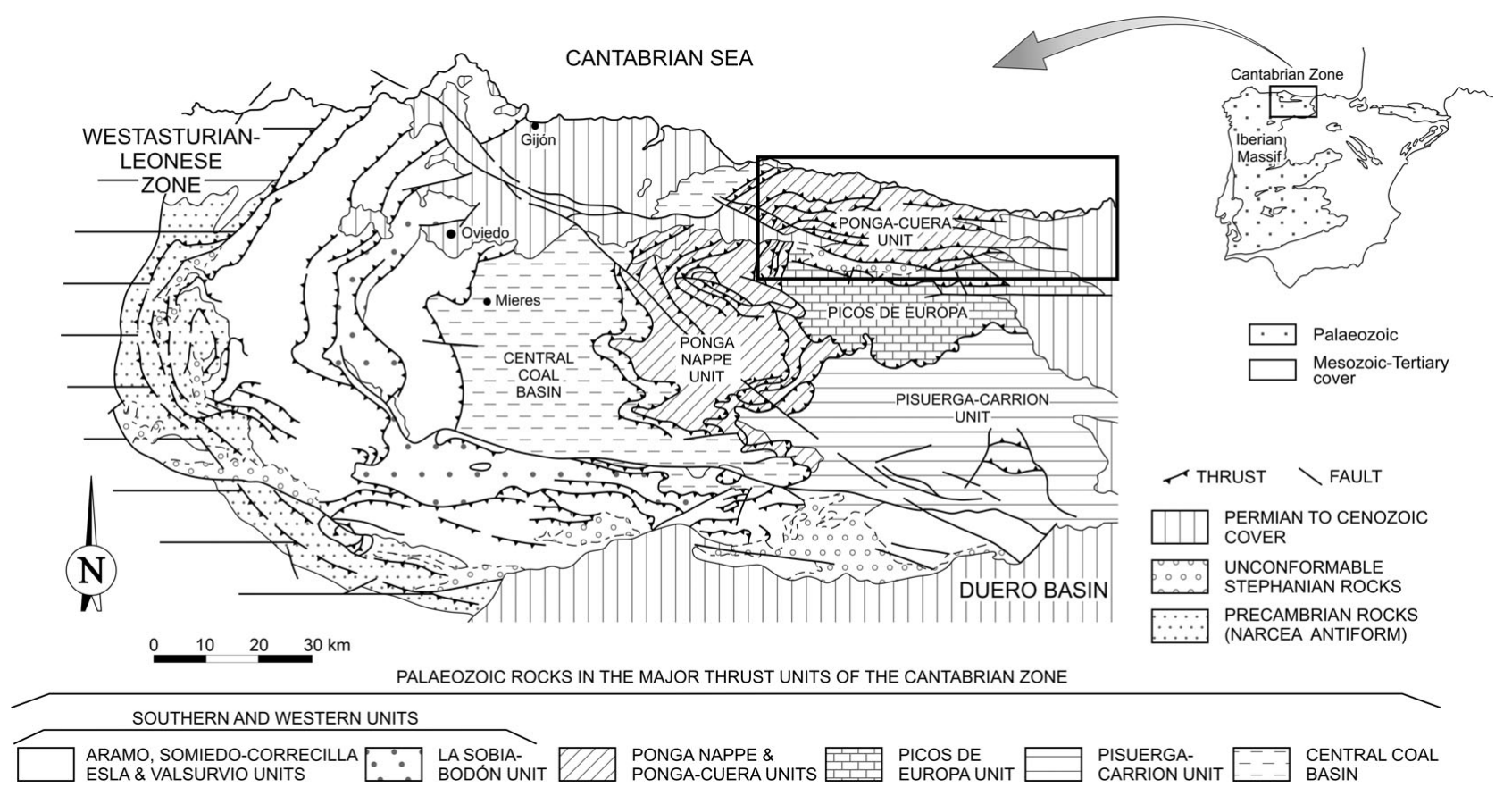

Figure 1. Generalized map of the Cantabrian Zone (after Julivert, 1971) with the location of the study area.

\section{Permian to Cenozoic cover of the Basque-Cantabrian basin.}

The Palaeozoic succession of the Ponga-Cuera unit is composed of two parts separated by an important stratigraphic gap (Fig. 2b). The lower part is composed of a lower-middle Cambrian carbonate formation and a Cambrian-Ordovician siliciclastic succession, with sandstone predominance; the thickness of this part of the succession is about $1000 \mathrm{~m}$. The upper part is a predominantly calcareous Carboniferous succession, although in the western part of the unit some siliciclastic levels appear due to lateral changes of facies. Locally, in the base of this part, a few metres of siliciclastic Devonian rocks crop out. The thickness of the Carboniferous succession is at least $2300 \mathrm{~m}$. Outside of the area studied, but near its southern boundary, an Pennsylvanian unconformable siliciclastic succession appears on the rocks described above. This succession contains carbonaceous shales and coal, the study of which provides pertinent data for the tectonothermal analysis of the area. In several areas, the Permian to Cenozoic rocks forming the cover lie unconformably on the Palaeozoic succession. The cover succession is composed of rocks with a local distribution and important sedimentary hiatuses. The estimated thickness of this cover is between 1300 and $2000 \mathrm{~m}$.

The structure of the Ponga-Cuera unit consists of a south-directed imbricate thrust system with a main décollement level in the Cambrian rocks (Fig. 2a, c). Some thrusts do not cut through the cover, and correspond to typical Variscan thrusts of the Cantabrian zone, but other thrusts cut through rocks of this cover and have been interpreted as Variscan thrusts that were reactivated during the Alpine deformation (Tosal, 1968).
The structural style of the study area is extended in the Picos de Europa unit. The two units are separated by a thrust with a large accumulated displacement (more than $20 \mathrm{~km}$ : Marquínez, 1989). In the western part of the Ponga-Cuera unit there are several NW-SE faults that displace the thrusts and have been interpreted as having originated during the Alpine deformation (Marquínez, 1989).

Ore deposits are common in the Ponga-Cuera unit (Fig. 3). They are mainly $\mathrm{Cu}, \mathrm{Co}-\mathrm{Ni}, \mathrm{Pb}-\mathrm{Zn}, \mathrm{Hg}-\mathrm{As}$, $\mathrm{Fe}-\mathrm{Mn}$, barite and fluorite mineralizations (MartínezGarcía, 1981, 1983; Luque \& Martínez-García, 1985; Martínez-García \& Tejerina, 1985; Martín-Izard, Paniagua \& Arias, 1995; Gutiérrez-Claverol \& Luque, 2000; Martínez-García et al. 2004; Luque \& GutiérrezClaverol, 2006). With the exception of some Mn deposits, which have been interpreted as syngenetic (Martínez-García et al. 2004), the rest of the deposits have been considered epigenetic and epithermal in origin, with formation temperatures between 100 and $150^{\circ} \mathrm{C}$ (Martín-Izard, Paniagua \& Arias, 1995), or $<200{ }^{\circ} \mathrm{C}$ (Loredo \& García Iglesias, 1981; Luque \& Martínez-García, 1985; Luque, Martínez-García \& Ruiz, 1990), and low pressure (20-60 MPa; Luque \& Martínez García, 1985; Luque, Martínez-García \& Ruiz, 1990). Most of them are hosted by Carboniferous limestones and have a strongly associated dolomitization and silicification. The minerals mainly appear in fractures or irregular bodies. Their origin has been related to the flow of hydrothermal fluids through fractures during a post-Variscan extensional episode. Field relationships and absolute dating in other similar ore deposits of the Cantabrian zone suggest a mainly Permian age for the ore deposits of the study area (Martínez García et al. 1991; Paniagua et al. 1993; Weh et al. 2001). 


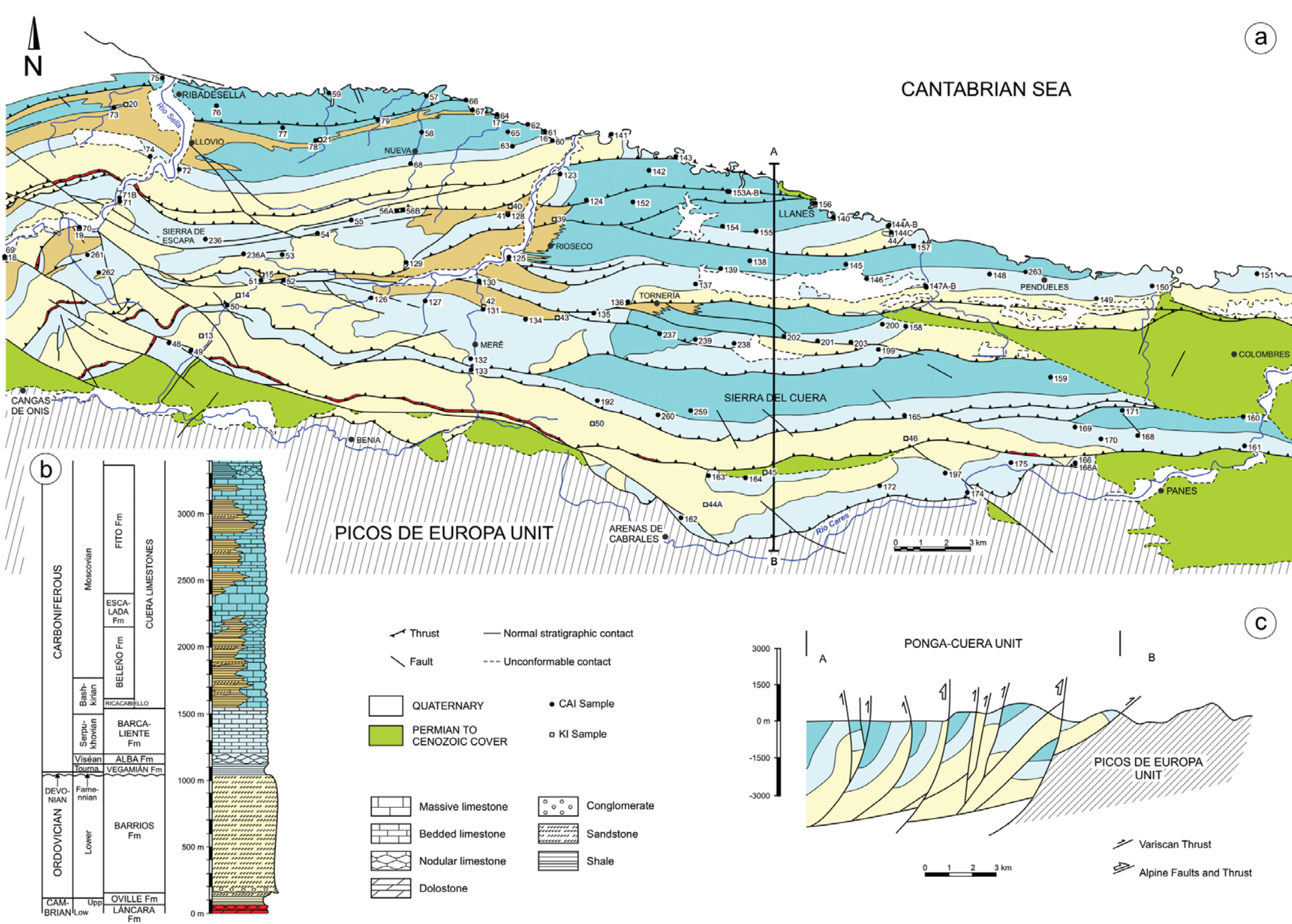




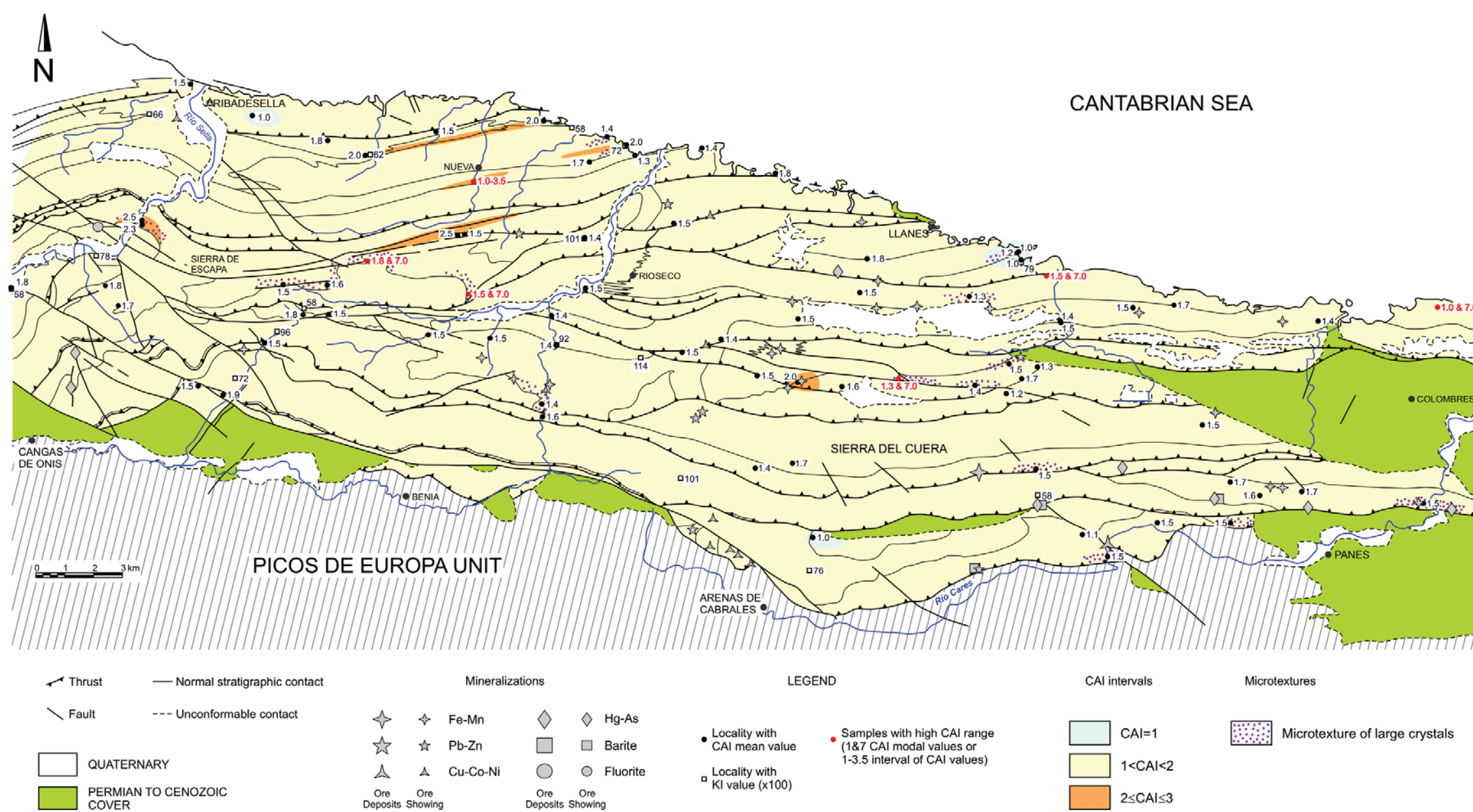

Figure 3. Map showing the distribution of colour alteration index (CAI) and Kübler index (KI) values, and the location of ore deposits and the microtexture of large crystals. 
Most of the Cantabrian zone is not metamorphic. None the less, the regional metamorphism, which is widespread in the hinterland, affects restricted areas in the western and northwestern parts of the Cantabrian zone, probably occurred in the Bashkirian-Moscovian, and involves cleavage development. In addition, an anchizonal or epizonal E-W band appears in the southern and southeastern parts of the Cantabrian zone. This band is associated with a nearly horizontal or moderately north-dipping cleavage that cuts across the main Variscan folds, and has been related to a late Variscan extensional episode that occurred near the Carboniferous-Permian boundary (García-López et al. 1999, 2007; Bastida et al. 2004). Hydrothermal activity related to normal faults continued during Permian and Mesozoic times.

\section{Materials and methods}

3.a. Conodont colour alteration index and textural analysis of these microfossils

More than one hundred samples were collected to obtain the CAI (Fig. 2, Table 1). The CAI values are based on specimens from limestone that were treated, 5-10 kg per sample, with 8-10\% buffered formic acid solution following the technique of Jeppsson \& Anehus (1995) for a short time (48 hours) to avoid etching or decolouration. The methodology used for CAI determination has been described in García-López et al. (1997) and Bastida et al. (1999). Information given by CAI data is restricted to the zones with outcrops of Carboniferous limestones, since the older Palaeozoic rocks do not contain conodonts. For the metamorphic zonation from the CAI data, we use the terms defined by García-López et al. (2001): diacaizone $(\mathrm{CAI}<4)$, ancaizone $(4 \leq \mathrm{CAI} \leq 5.5)$ and epicaizone $(\mathrm{CAI}>5.5)$. At each locality, the mean CAI value has been used for contouring the CAI data on the map. Temperature ranges of the CAI values can be obtained from the Arrhenius plot presented by Epstein, Epstein \& Harris (1977) and Rejebian, Harris \& Huebner (1987). Variations on the CAI values within a sample, and from sample to sample, within a small area, together with conodont texture, can help to distinguish grades and types of metamorphism (Rejebian, Harris \& Huebner, 1987).

It is common for conodonts from a single sample to have dispersed CAI values. Dispersion ranges $\leq 1$ are due to normal variability in the amount of organic matter of the different morphotypes of the sample, and the mean CAI values have been used in these cases in order to plot CAI data on the map. When the dispersion range is $>1$, and a single CAI modal interval exists, the presence of fluids is probable. In these cases, the CAI values of the two range extremes have been represented on the map (Fig. 3). A range of $>1$, with two CAI separate distributions and their corresponding modal values, is always due to fluid processes; in these cases two mean values have been determined and represented on the map. The existence of cases with a high dispersion of CAI values, sometimes with bimodal distributions, cannot be used to assess palaeotemperatures (Rejebian, Harris \& Huebner, 1987).

Besides the colour changes, conodonts underwent textural transformations on their surface that can provide interesting information about the physical and chemical environment conditions where they appear. Systematic observation of conodonts under the optical and scanning electron microscope has been carried out in order to analyse the textural features and infer the processes involved in their development. In this way, one to fifteen conodont elements from each positive sample were observed under the scanning electron microscope. Throughout this paper, we use the term 'texture' to refer to surface features of the conodonts at the scale of the optical microscope and the term 'microtexture' to refer these features at the scale of the electron microscope.

\section{3.b. Layer silicate mineralogy and Kübler index}

Sixteen samples of pelitic rock were collected for Xray diffraction analyses in order to determine their phyllosilicate mineralogy and the Kübler Index (KI) of illite (see Guggenheim et al. 2002) (Fig. 2, Table 2). The clay fraction $<2 \mu \mathrm{m}$ was separated by centrifugation. Oriented aggregates were prepared by sedimentation on glass slides and air-dried in atmospheric conditions. The slides were then saturated with ethylene glycol and heated at 300 and $550{ }^{\circ} \mathrm{C}$. Xray diffraction patterns were obtained using a Philips automated PW3200 X'Pert X-ray diffractometer, $\mathrm{CuK}_{\alpha}$ radiation, and a graphite monochromator operating at $40 \mathrm{kV}$ and $30 \mathrm{~mA}$, with intervals of $0.02^{\circ} 2 \theta$ for at least $2 \mathrm{~s}$. Preparation of samples and determination of the Kübler Index were done taking into account the recommendation of the IGCP 294 working group (Kisch, 1991). The KI values obtained were converted to the Kübler scale with anchizone limits of $0.42^{\circ} / 0.25^{\circ}$ $\Delta 2 \theta$, using a set of samples provided by H. J. Kisch (Ben-Gurion University of Negev, Israel). The KI values have been converted to the Kübler scale using the calibration equation:

$$
\mathrm{KI}_{\text {Kübler }}=0.805 \mathrm{KI}_{\text {Oviedo }}+0.096\left(\mathrm{R}^{2}=0.971\right)
$$

\section{Results}

In the study area, most of the CAI values range from 1 to 2 (Fig. 3), indicating a temperature interval from 50 to $80{ }^{\circ} \mathrm{C}$ (Table 1 ). Values of $2 \leq \mathrm{CAI} \leq 3$ appear only in very local areas and they are taken as anomalous values with respect to the general background. In some cases, conodonts from the same locality present a high dispersion of CAI values and two modal values, one with a value between 1 and 2 and another one with 
Table 1. Conodont colour alteration index (CAI) values and temperatures inferred from the CAI Arrhenius plot (Epstein, Epstein \& Harris, 1977; Rejebian, Harris \& Huebner, 1987) according to the heating interval of the rocks between one and one hundred million of years; selected characteristics of the texture and the microtexture of the conodonts from the Ponga-Cuera unit are indicated

\begin{tabular}{|c|c|c|c|c|c|c|c|c|}
\hline $\begin{array}{l}\text { Locality } \\
\text { number }\end{array}$ & Formation & Age & $\begin{array}{l}\text { CAI (number } \\
\text { of specimens) }\end{array}$ & $\begin{array}{l}\text { Mean } \\
\text { value }\end{array}$ & $\begin{array}{l}\text { Standard } \\
\text { deviation }\end{array}$ & $\begin{array}{l}\text { Temperature } \\
\text { range }\left({ }^{\circ} \mathrm{C}\right)\end{array}$ & Texture (OM) & Microtexture (SEM) \\
\hline 48 & Barcaliente & Serpukhovian & $1.5(1)$ & 1.5 & - & $<50-57$ & $\begin{array}{l}\text { sugary, bleached, } \\
\text { grey patina }\end{array}$ & - \\
\hline 49 & Barcaliente & Serpukhovian & $1.5(4) ; 2(12)$ & 1.9 & 0.22 & $<50-75$ & $\begin{array}{l}\text { smooth, sugary, } \\
\text { bleached }\end{array}$ & $\begin{array}{l}\text { small crystals, } \\
\text { irregular envelope }\end{array}$ \\
\hline 50 & Barcaliente & Serpukhovian & $1.5(4)$ & 1.5 & - & $<50-57$ & sugary, bleached & $\begin{array}{l}\text { small crystals, } \\
\text { external casts, } \\
\text { dissolution, } \\
\text { irregular envelope }\end{array}$ \\
\hline 51 & Barcaliente & Serpukhovian & $\begin{array}{c}1.5(2) \\
1.5-2(2) ; 2(4)\end{array}$ & 1.8 & 0.22 & $<50-70$ & $\begin{array}{l}\text { sugary, bleached, } \\
\text { grey patina, } \\
\text { filling of basal } \\
\text { cavity }\end{array}$ & $\begin{array}{l}\text { small crystals, } \\
\text { external casts }\end{array}$ \\
\hline 52 & Barcaliente & Serpukhovian & $1.5(9)$ & 1.5 & - & $<50-57$ & $\begin{array}{l}\text { smooth, sugary, } \\
\text { bleached, grey } \\
\text { patina, filling of } \\
\text { basal cavity }\end{array}$ & $\begin{array}{l}\text { small crystals, } \\
\text { external casts, } \\
\text { irregular envelope }\end{array}$ \\
\hline 53 & Alba & Viséan & $\begin{array}{c}1(3) ; 1.5(123) ; \\
2(63)\end{array}$ & 1.6 & 0.25 & $<50-60$ & $\begin{array}{l}\text { sugary, bleached, } \\
\text { pink patina, } \\
\text { filling of basal } \\
\text { cavity }\end{array}$ & $\begin{array}{l}\text { small crystals, } \\
\text { external casts, } \\
\text { large crystals, } \\
\text { corrosion, } \\
\text { irregular envelope }\end{array}$ \\
\hline 54 & Barcaliente & Serpukhovian & $1.5-2(2) ; 7(5)$ & $\begin{array}{l}1.8 \& \\
7.0\end{array}$ & - & - & $\begin{array}{l}\text { sugary, grey patina, } \\
\text { filling of basal } \\
\text { cavity. CAI =7 } \\
\text { very bleached }\end{array}$ & $\begin{array}{l}\text { small crystals, } \\
\text { external cast, } \\
\text { corrosion, } \\
\text { irregular envelope }\end{array}$ \\
\hline $56 \mathrm{~A}$ & Alba & Viséan & $2(1) ; 3(1)$ & 2.5 & 0.70 & & sugary & $\begin{array}{l}\text { small crystals, } \\
\quad \text { irregular envelope }\end{array}$ \\
\hline $56 \mathrm{~B}$ & Barcaliente & Serpukhovian & $1.5(3)$ & 1.5 & - & $<50-57$ & sugary, bleached & $\begin{array}{l}\text { small crystals, } \\
\text { external casts, } \\
\text { irregular envelope }\end{array}$ \\
\hline 60 & Barcaliente & Serpukhovian & $1(3) ; 1.5(4)$ & 1.3 & 0.26 & $<55$ & $\begin{array}{l}\text { sugary, bleached, } \\
\text { grey patina, } \\
\text { filling of basal } \\
\text { cavity (Q } \\
\text { crystals) }\end{array}$ & $\begin{array}{l}\text { small crystals, } \\
\text { external casts }\end{array}$ \\
\hline 61 & $\begin{array}{l}\text { Cuera Limestones } \\
\quad \text { (lower) }\end{array}$ & Bashkirian & $2(40)$ & 2.0 & - & $50-80$ & $\begin{array}{l}\text { sugary, grey patina, } \\
\text { filling of basal } \\
\text { cavity (Q } \\
\text { crystals) }\end{array}$ & $\begin{array}{l}\text { small crystals, } \\
\text { external casts, } \\
\text { large crystals, } \\
\text { corrosion, } \\
\text { irregular envelope }\end{array}$ \\
\hline 62 & $\begin{array}{l}\text { Cuera Limestones } \\
\quad \text { (upper) }\end{array}$ & Moscovian & $\begin{array}{c}1(104) ; \\
1.5(233) ; 2(44)\end{array}$ & 1.4 & 0.30 & $<55$ & $\begin{array}{l}\text { smooth, sugary, } \\
\text { bleached, grey } \\
\text { patina, filling of } \\
\text { basal cavity }\end{array}$ & $\begin{array}{l}\text { small crystals, } \\
\text { external casts, } \\
\text { large crystals, } \\
\text { irregular envelope }\end{array}$ \\
\hline 63 & $\begin{array}{l}\text { Cuera Limestones } \\
\text { (lower) }\end{array}$ & Bashkirian & $1.5(7) ; 2(3)$ & 1.7 & 0.25 & $<50-65$ & sugary, grey patina & $\begin{array}{l}\text { small crystals, } \\
\text { external casts, } \\
\text { irregular envelope }\end{array}$ \\
\hline 67 & $\begin{array}{l}\text { Cuera Limestones } \\
\text { (upper) }\end{array}$ & Moscovian & $2(1)$ & 2.0 & - & $50-80$ & sugary, grey patina & $\begin{array}{l}\text { corrosion, irregular } \\
\text { envelope }\end{array}$ \\
\hline 68 & Barcaliente & Serpukhovian & $\begin{array}{l}1(1) ; 1.5(3) ; \\
2.5(2) ; 3(2) ; \\
\quad 3.5(1)\end{array}$ & $1-3.5$ & - & - & $\begin{array}{l}\text { sugary, bleached, } \\
\text { grey patina, } \\
\text { filling of basal } \\
\text { cavity }\end{array}$ & $\begin{array}{l}\text { external casts, } \\
\text { corrosion, } \\
\text { irregular envelope }\end{array}$ \\
\hline 69 & Barcaliente & Serpukhovian & $1.5(1) ; 2(2)$ & 1.8 & 0.28 & $50-70$ & $\begin{array}{l}\text { sugary, bleached, } \\
\text { grey patina, } \\
\text { filling of basal } \\
\text { cavity (Q } \\
\text { crystals) }\end{array}$ & - \\
\hline 71 & Alba & Viséan & $2(1) ; 2.5(1)$ & 2.3 & 0.35 & $65-100$ & sugary & $\begin{array}{l}\text { large crystals, } \\
\text { corrosion, } \\
\text { irregular envelope }\end{array}$ \\
\hline 71B & Alba & Viséan & $2.5(1)$ & 2.5 & - & $75-115$ & sugary & $\begin{array}{l}\text { small crystals, } \\
\text { external casts, } \\
\text { irregular envelope }\end{array}$ \\
\hline 75 & $\begin{array}{l}\text { Cuera Limestones } \\
\text { (lower) }\end{array}$ & Bashkirian & $1.5(5)$ & 1.5 & - & $<50-57$ & $\begin{array}{l}\text { sugary, bleached, } \\
\text { corrosion, grey } \\
\text { patina, filling of } \\
\text { basal cavity }\end{array}$ & $\begin{array}{l}\text { external casts, } \\
\text { corrosion, } \\
\text { irregular envelope }\end{array}$ \\
\hline 76 & $\begin{array}{l}\text { Cuera Limestones } \\
\text { (lower) }\end{array}$ & Bashkirian & $1(2)$ & 1.0 & - & $<55$ & $\begin{array}{l}\text { sugary, bleached, } \\
\text { grey patina }\end{array}$ & - \\
\hline 77 & $\begin{array}{l}\text { Cuera Limestones } \\
\text { (upper) }\end{array}$ & Moscovian & $1.5(2) ; 2(2)$ & 1.8 & 0.28 & $50-70$ & $\begin{array}{l}\text { sugary, bleached, } \\
\text { grey patina }\end{array}$ & - \\
\hline
\end{tabular}


Table 1. Continued.

\begin{tabular}{|c|c|c|c|c|c|c|c|c|}
\hline $\begin{array}{l}\text { Locality } \\
\text { number }\end{array}$ & Formation & Age & $\begin{array}{l}\text { CAI (number } \\
\text { of specimens) }\end{array}$ & $\begin{array}{l}\text { Mean } \\
\text { value }\end{array}$ & $\begin{array}{l}\text { Standard } \\
\text { deviation }\end{array}$ & $\begin{array}{c}\text { Temperature } \\
\text { range }\left({ }^{\circ} \mathrm{C}\right)\end{array}$ & Texture (OM) & Microtexture (SEM) \\
\hline 78 & $\begin{array}{l}\text { Cuera Limestones } \\
\text { (upper) }\end{array}$ & Moscovian & $2(1)$ & 2.0 & - & $50-80$ & sugary & $\begin{array}{l}\text { small crystals, } \\
\text { external casts, } \\
\text { irregular envelope }\end{array}$ \\
\hline 79 & $\begin{array}{l}\text { Cuera Limestones } \\
\text { (upper) }\end{array}$ & Moscovian & $1.5(8)$ & 1.5 & - & $<50-57$ & $\begin{array}{l}\text { sugary, bleached, } \\
\text { grey patina, } \\
\text { filling of basal } \\
\text { cavity (Q } \\
\text { crystals) }\end{array}$ & $\begin{array}{l}\text { small crystals, } \\
\text { corrosion, } \\
\text { irregular envelope }\end{array}$ \\
\hline 124 & $\begin{array}{l}\text { Cuera Limestones } \\
\text { (lower) }\end{array}$ & Bashkirian & $1.5(5)$ & 1.5 & - & $<50-57$ & $\begin{array}{l}\text { sugary, bleached, } \\
\text { grey patina }\end{array}$ & $\begin{array}{l}\text { small crystals, } \\
\text { external casts, } \\
\text { corrosion }\end{array}$ \\
\hline 125 & Barcaliente & Serpukhovian & $1.5(1)$ & 1.5 & - & $<50-57$ & sugary & $\begin{array}{l}\text { small crystals, } \\
\text { external casts, } \\
\text { irregular envelope }\end{array}$ \\
\hline 126 & Barcaliente & Serpukhovian & $1.5(1)$ & 1.5 & - & $<50-57$ & sugary, bleached & - \\
\hline 127 & Barcaliente & Serpukhovian & $1.5(3)$ & 1.5 & - & $<50-57$ & $\begin{array}{l}\text { sugary, bleached, } \\
\text { filling of basal } \\
\text { cavity (Q } \\
\text { crystals) }\end{array}$ & $\begin{array}{l}\text { small crystals, } \\
\text { external casts }\end{array}$ \\
\hline 128 & Beleño & $\begin{array}{l}\text { Lower } \\
\text { Moscovian }\end{array}$ & $1(1) ; 1.5(5)$ & 1.4 & 0.20 & $<55$ & $\begin{array}{l}\text { smooth, sugary, } \\
\text { bleached, grey } \\
\text { patina }\end{array}$ & $\begin{array}{l}\text { small crystals, } \\
\text { external casts, } \\
\text { corrosion, } \\
\text { irregular envelope }\end{array}$ \\
\hline 129 & Alba & Viséan & $\begin{array}{l}1(7) ; 1.5(42) \\
2(2) ; 7(1)\end{array}$ & $\begin{array}{l}1.5 \& \\
7.0\end{array}$ & - & - & $\begin{array}{l}\text { sugary, bleached, } \\
\text { grey patina }\end{array}$ & $\begin{array}{l}\text { small crystals, } \\
\text { external casts, } \\
\text { large crystals, } \\
\text { corrosion, } \\
\text { irregular envelope }\end{array}$ \\
\hline 130 & Barcaliente & Serpukhovian & $\begin{array}{l}1(4) ; 1.5(6) ; \\
2(1)\end{array}$ & 1.4 & 0.32 & $<55$ & $\begin{array}{l}\text { smooth, sugary, } \\
\text { bleached }\end{array}$ & $\begin{array}{l}\text { small crystals, } \\
\text { corrosion, } \\
\text { irregular envelope }\end{array}$ \\
\hline 131 & $\begin{array}{r}\text { Ricacabiello- } \\
\text { Barcaliente }\end{array}$ & Serpukhovian & $\begin{array}{c}1(24) ; 1.5(24) ; \\
2(10)\end{array}$ & 1.4 & 0.36 & $<55$ & $\begin{array}{l}\text { smooth, sugary, } \\
\text { bleached, grey } \\
\text { patina }\end{array}$ & $\begin{array}{l}\text { small crystals, } \\
\text { external casts, } \\
\text { irregular envelope }\end{array}$ \\
\hline 132 & Alba & Viséan & $1(12) ; 1.5(46)$ & 1.4 & 0.20 & $<55$ & $\begin{array}{l}\text { sugary, bleached, } \\
\text { grey patina }\end{array}$ & $\begin{array}{l}\text { small crystals, } \\
\text { external casts, } \\
\text { large crystals, } \\
\text { corrosion, } \\
\text { irregular envelope }\end{array}$ \\
\hline 133 & Barcaliente & Serpukhovian & $\begin{array}{c}1(29) ; 1.5(6) \\
2(3)\end{array}$ & 1.6 & 0.35 & $<50-60$ & $\begin{array}{l}\text { sugary, bleached, } \\
\text { corrosion }\end{array}$ & $\begin{array}{l}\text { small crystals, } \\
\text { external casts, } \\
\text { corrosion }\end{array}$ \\
\hline 135 & Barcaliente & Serpukhovian & $1.5(1)$ & 1.5 & - & $<50-57$ & $\begin{array}{l}\text { sugary, bleached, } \\
\text { corrosion }\end{array}$ & $\begin{array}{l}\text { unalterated, small } \\
\text { crystals, external } \\
\text { casts, corrosion, } \\
\text { irregular envelope }\end{array}$ \\
\hline 136 & Barcaliente & Serpukhovian & $1(2) ; 1.5(5)$ & 1.4 & 0.24 & $<55$ & $\begin{array}{l}\text { smooth, sugary, } \\
\text { bleached }\end{array}$ & $\begin{array}{l}\text { small crystals, } \\
\text { external casts, } \\
\text { corrosion, } \\
\text { irregular envelope }\end{array}$ \\
\hline 137 & Barcaliente & Serpukhovian & $1.5(5)$ & 1.5 & - & $<50-57$ & sugary, bleached & $\begin{array}{l}\text { small crystals, } \\
\text { external casts }\end{array}$ \\
\hline 138 & $\begin{array}{l}\text { Cuera Limestones } \\
\text { (lower) }\end{array}$ & Bashkirian & $1.5(9)$ & 1.5 & - & $<50-57$ & sugary, bleached & $\begin{array}{l}\text { small crystals, } \\
\text { external casts, } \\
\text { corrosion, } \\
\text { irregular envelope }\end{array}$ \\
\hline 141 & Barcaliente & Serpukhovian & $1(1) ; 1.5(4)$ & 1.4 & 0.22 & $<55$ & $\begin{array}{l}\text { smooth, sugary, } \\
\text { bleached }\end{array}$ & $\begin{array}{l}\text { small crystals, } \\
\text { external casts, } \\
\text { corrosion, } \\
\text { irregular envelope }\end{array}$ \\
\hline 143 & Barcaliente & hovian & $1.5-2(1)$ & 1.8 & - & $50-70$ & - & - \\
\hline 144A & Alba & Viséan & $\begin{array}{c}1(17) ; 1.5(3) \\
2(2)\end{array}$ & 1.2 & 0.32 & $<5$ & $\begin{array}{l}\text { sugary, bleached, } \\
\text { grey patina }\end{array}$ & $\begin{array}{l}\text { small crystals, large } \\
\text { crystals, } \\
\text { corrosion, } \\
\text { irregular envelope }\end{array}$ \\
\hline 144B & Alba & Viséan & 1(1) & 1.0 & - & $<55$ & $\begin{array}{l}\text { smooth, sugary, } \\
\text { bleached }\end{array}$ & - \\
\hline $144 \mathrm{C}$ & Alba & Viséan & 1(2) & 1.0 & - & $<55$ & sugary, bleached & - \\
\hline 145 & $\begin{array}{l}\text { Cuera Limestones } \\
\text { (upper) }\end{array}$ & Moscovian & $1(1) ; 1.5(2)$ & 1.3 & 0.28 & $<55$ & $\begin{array}{l}\text { sugary, bleached, } \\
\text { grey patina }\end{array}$ & $\begin{array}{l}\text { small crystals, } \\
\text { external casts, } \\
\text { large crystals, } \\
\text { corrosion, } \\
\text { irregular envelope }\end{array}$ \\
\hline
\end{tabular}


Table 1. Continued.

\begin{tabular}{|c|c|c|c|c|c|c|c|c|}
\hline $\begin{array}{l}\text { Locality } \\
\text { number }\end{array}$ & Formation & Age & $\begin{array}{l}\text { CAI (number } \\
\text { of specimens) }\end{array}$ & $\begin{array}{l}\text { Mean } \\
\text { value }\end{array}$ & $\begin{array}{l}\text { Standard } \\
\text { deviation }\end{array}$ & $\begin{array}{l}\text { Temperature } \\
\text { range }\left({ }^{\circ} \mathrm{C}\right)\end{array}$ & Texture (OM) & Microtexture (SEM) \\
\hline $147 \mathrm{~A}$ & Alba & Viséan & $1(4) ; 1.5(11)$ & 1.4 & 0.22 & $<55$ & $\begin{array}{l}\text { smooth, sugary, } \\
\text { bleached }\end{array}$ & $\begin{array}{l}\text { small crystals, } \\
\text { external casts, } \\
\text { corrosion, } \\
\text { irregular envelope }\end{array}$ \\
\hline 147B & Alba & Viséan & $1.5(22)$ & 1.5 & - & $<50-57$ & $\begin{array}{l}\text { sugary, bleached, } \\
\text { grey patina, } \\
\text { filling of basal } \\
\text { cavity }\end{array}$ & $\begin{array}{l}\text { small crystals, } \\
\text { external casts, } \\
\text { irregular envelope }\end{array}$ \\
\hline 148 & Barcaliente & Serpukhovian & $1.5(1)$ & 1.5 & - & $<50-57$ & sugary & external casts \\
\hline 150 & Barcaliente & Serpukhovian & $\begin{array}{c}1(5) ; 1.5(15) \\
2(1)\end{array}$ & 1.4 & 0.25 & $<55$ & sugary, bleached & $\begin{array}{l}\text { small crystals, } \\
\text { external casts, } \\
\text { irregular envelope }\end{array}$ \\
\hline 151 & Barcaliente & Serpukhovian & $1(1) ; 7(1)$ & $\begin{array}{c}1.0 \& \\
7.0\end{array}$ & - & - & $\begin{array}{l}\text { sugary, bleached. } \\
\text { CAI = } 7 \text { very } \\
\text { bleached }\end{array}$ & - \\
\hline 155 & $\begin{array}{l}\text { Cuera Limestones } \\
\text { (lower) }\end{array}$ & Bashkirian & $\begin{array}{l}1.5(34) \\
1.5-2(85) \\
2(87)\end{array}$ & 1.8 & 0.18 & $50-70$ & $\begin{array}{l}\text { sugary, very } \\
\text { sugary, bleached, } \\
\text { grey patina, } \\
\text { corroded, filling } \\
\text { of basal cavity } \\
\text { (Q crystals) }\end{array}$ & $\begin{array}{l}\text { small crystals, } \\
\text { external casts, } \\
\text { corrosion, } \\
\text { irregular envelope }\end{array}$ \\
\hline 157 & $\begin{array}{l}\text { Cuera Limestones } \\
\text { (upper) }\end{array}$ & Moscovian & $\begin{array}{c}1(1) ; 1.5(12) \\
7(2)\end{array}$ & $\begin{array}{l}1.5 \& \\
7.0\end{array}$ & - & - & $\begin{array}{l}\text { sugary, bleached, } \\
\text { grey patina, } \\
\text { corroded. CAI = } \\
7 \text { very bleached }\end{array}$ & $\begin{array}{l}\text { small crystals, } \\
\text { external casts, } \\
\text { corrosion, } \\
\text { irregular envelope }\end{array}$ \\
\hline 158 & Barcaliente & Serpukhovian & $1(1) ; 1.5(1)$ & 1.3 & 0.35 & $<55$ & $\begin{array}{l}\text { smooth, sugary, } \\
\text { bleached }\end{array}$ & $\begin{array}{l}\text { small crystals, } \\
\text { external casts, } \\
\text { irregular envelope, } \\
\text { corrosion }\end{array}$ \\
\hline 159 & $\begin{array}{l}\text { Cuera Limestones } \\
\text { (lower) }\end{array}$ & Bashkirian & $1.5(45) ; 2(2)$ & 1.5 & 0.10 & $<50-57$ & $\begin{array}{l}\text { smooth, sugary, } \\
\text { bleached, grey } \\
\text { patina }\end{array}$ & $\begin{array}{l}\text { small crystals, } \\
\text { external casts, } \\
\text { corrosion, } \\
\text { irregular envelope }\end{array}$ \\
\hline 161 & Barcaliente & Serpukhovian & $1.5(6)$ & 1.5 & - & $<50-57$ & $\begin{array}{l}\text { sugary, bleached, } \\
\text { grey patina }\end{array}$ & $\begin{array}{l}\text { small crystals, } \\
\text { external casts }\end{array}$ \\
\hline 163 & Alba & Viséan & $1(26) ; 1.5(2)$ & 1.0 & 0.13 & $<55$ & $\begin{array}{l}\text { sugary, bleached, } \\
\text { grey patina }\end{array}$ & $\begin{array}{l}\text { large crystals, } \\
\quad \text { irregular envelope }\end{array}$ \\
\hline 165 & Alba & Viséan & $1.5(15)$ & 1.5 & - & $<50-57$ & $\begin{array}{l}\text { sugary, bleached, } \\
\text { grey patina }\end{array}$ & $\begin{array}{l}\text { small crystals, large } \\
\text { crystals, irregular } \\
\text { envelope }\end{array}$ \\
\hline 166 & Alba & Viséan & $1.5(91) ; 2(4)$ & 1.5 & 0.10 & $<50-57$ & $\begin{array}{l}\text { sugary, bleached, } \\
\text { grey and pink } \\
\text { patina, filling of } \\
\text { basal cavity }\end{array}$ & $\begin{array}{l}\text { small crystals, large } \\
\text { crystals, } \\
\text { corrosion, } \\
\text { irregular envelope }\end{array}$ \\
\hline 168 & Barcaliente & Serpukhovian & $1.5(15) ; 2(9)$ & 1.7 & 0.24 & $<50-65$ & $\begin{array}{l}\text { sugary, very } \\
\text { sugary, grey } \\
\text { patina, corroded, } \\
\text { filling of basal } \\
\text { cavity }\end{array}$ & $\begin{array}{l}\text { small crystals, } \\
\text { external casts, } \\
\text { large crystals, } \\
\text { corrosion, } \\
\text { irregular envelope }\end{array}$ \\
\hline 169 & Barcaliente & Serpukhovian & $\begin{array}{c}1(3) ; 1.5(1) \\
2(7)\end{array}$ & 1.7 & 0.46 & $<50-65$ & $\begin{array}{l}\text { smooth, sugary, } \\
\text { bleached }\end{array}$ & - \\
\hline 170 & Barcaliente & Serpukhovian & $\begin{array}{c}1.5(9) \\
1.5-2(5)\end{array}$ & 1.6 & 0.12 & $<50-60$ & $\begin{array}{l}\text { smooth, sugary, } \\
\text { bleached }\end{array}$ & $\begin{array}{l}\text { small crystals, } \\
\text { external casts, } \\
\text { corrosion, } \\
\text { irregular envelope }\end{array}$ \\
\hline 174 & Valdeteja & Bashkirian & $1.5(30)$ & 1.5 & - & $<50-57$ & $\begin{array}{l}\text { sugary, very } \\
\text { sugary, bleached, } \\
\text { grey patina }\end{array}$ & $\begin{array}{l}\text { small crystals, } \\
\text { external casts, } \\
\text { large crystals, } \\
\text { corrosion, } \\
\text { irregular envelope }\end{array}$ \\
\hline 175 & Barcaliente & Serpukhovian & $1.5(3)$ & 1.5 & - & $<50-57$ & sugary, bleached & $\begin{array}{l}\text { small crystals, } \\
\text { external casts, } \\
\text { corrosion, } \\
\text { irregular envelope }\end{array}$ \\
\hline 197 & Valdeteja & Bashkirian & $1(6) ; 1.5(2)$ & 1.1 & 0.23 & $<55$ & $\begin{array}{l}\text { sugary, very } \\
\text { sugary, bleached, } \\
\text { grey patina. } \\
\text { CAI = } 1 \text { very } \\
\text { bleached }\end{array}$ & $\begin{array}{l}\text { small crystals, } \\
\text { external casts, } \\
\text { irregular envelope }\end{array}$ \\
\hline 199 & Barcaliente & Serpukhovian & $1(2) ; 1.5(1)$ & 1.2 & 0.28 & $<55$ & $\begin{array}{l}\text { smooth, sugary, } \\
\text { bleached }\end{array}$ & $\begin{array}{l}\text { corrosion, external } \\
\text { casts, small } \\
\text { crystals }\end{array}$ \\
\hline 200 & $\begin{array}{l}\text { Cuera Limestones } \\
\text { (lower) }\end{array}$ & Bashkirian & $\begin{array}{l}1(1) ; 1.5(14) \\
1.5-2(3)\end{array}$ & 1.5 & 0.15 & $<50-57$ & $\begin{array}{l}\text { sugary, bleached, } \\
\text { grey patina, } \\
\text { filling of basal } \\
\text { cavity }\end{array}$ & $\begin{array}{l}\text { small crystals, } \\
\text { external casts, } \\
\text { large crystals, } \\
\text { irregular envelope }\end{array}$ \\
\hline
\end{tabular}


Table 1. Continued.

\begin{tabular}{|c|c|c|c|c|c|c|c|c|}
\hline $\begin{array}{l}\text { Locality } \\
\text { number }\end{array}$ & Formation & Age & $\begin{array}{l}\text { CAI (number } \\
\text { of specimens) }\end{array}$ & $\begin{array}{l}\text { Mean } \\
\text { value }\end{array}$ & $\begin{array}{l}\text { Standard } \\
\text { deviation }\end{array}$ & $\begin{array}{l}\text { Temperature } \\
\text { range }\left({ }^{\circ} \mathrm{C}\right)\end{array}$ & Texture (OM) & Microtexture (SEM) \\
\hline 202 & Alba & Viséan & $\begin{array}{c}1(8) ; 1.5(4) ; \\
2(3) ; 7(1)\end{array}$ & $\begin{array}{l}1.3 \& \\
7.0\end{array}$ & - & - & $\begin{array}{l}\text { sugary, bleached, } \\
\text { grey patina, } \\
\text { filling of basal } \\
\text { cavity (Q } \\
\text { crystals). CAI = } \\
1 \text { and CAI }=7 \\
\text { very bleached }\end{array}$ & $\begin{array}{l}\text { small crystals, large } \\
\text { crystals, } \\
\text { corrosion, external } \\
\text { casts, irregular } \\
\text { envelope }\end{array}$ \\
\hline 203 & Alba & Viséan & $\begin{array}{c}1(54) ; 1.5(84) \\
2(11)\end{array}$ & 1.4 & 0.29 & $<55$ & $\begin{array}{l}\text { sugary, bleached, } \\
\text { pink patina }\end{array}$ & $\begin{array}{l}\text { small crystals, } \\
\text { external casts, } \\
\text { large crystals, } \\
\text { corrosion, } \\
\text { irregular envelope }\end{array}$ \\
\hline $236 \mathrm{~A}$ & Alba & Viséan & $1.5(2)$ & 1.5 & - & $<50-57$ & sugary, bleached & $\begin{array}{l}\text { small crystals, } \\
\text { irregular envelope, } \\
\text { large crystals }\end{array}$ \\
\hline 237 & $\begin{array}{l}\text { Cuera Limestones } \\
\quad \text { (upper) }\end{array}$ & Moscovian & $1(1) ; 1.5(16)$ & 1.5 & 0.12 & $<50-57$ & sugary, bleached & $\begin{array}{l}\text { small crystals, } \\
\text { external casts, } \\
\text { corrosion, } \\
\text { irregular envelope }\end{array}$ \\
\hline 238 & $\begin{array}{l}\text { Cuera Limestones } \\
\text { (upper) }\end{array}$ & Moscovian & $\begin{array}{c}1(3) ; 1.5(34) ; \\
2(13)\end{array}$ & 1.6 & 0.26 & $<50-60$ & $\begin{array}{l}\text { smooth, sugary, } \\
\text { bleached, grey } \\
\text { patina }\end{array}$ & $\begin{array}{l}\text { small crystals, } \\
\text { external casts, } \\
\text { corrosion, } \\
\text { irregular envelope }\end{array}$ \\
\hline 239 & $\begin{array}{l}\text { Cuera Limestones } \\
\text { (upper) }\end{array}$ & Moscovian & $2(2)$ & 2.0 & - & $50-80$ & $\begin{array}{l}\text { sugary, bleached, } \\
\text { grey patina }\end{array}$ & $\begin{array}{l}\text { small crystals, } \\
\text { external casts, } \\
\text { irregular envelope }\end{array}$ \\
\hline 259 & $\begin{array}{l}\text { Cuera Limestones } \\
\quad \text { (lower) }\end{array}$ & Bashkirian & $\begin{array}{c}1.5(2) ; \\
1.5-2(10)\end{array}$ & 1.7 & 0.10 & $<50-65$ & $\begin{array}{l}\text { sugary, bleached, } \\
\text { grey patina }\end{array}$ & $\begin{array}{l}\text { small crystals, } \\
\text { external casts, } \\
\text { corrosion, } \\
\text { irregular envelope }\end{array}$ \\
\hline 260 & Barcaliente & Serpukhovian & 1(12); 1.5(29) & 1.4 & 0.23 & $<55$ & $\begin{array}{l}\text { smooth, sugary, } \\
\text { bleached, grey } \\
\text { patina, filling of } \\
\text { basal cavity }\end{array}$ & $\begin{array}{l}\text { small crystals, } \\
\text { external casts, } \\
\text { corrosion, } \\
\text { irregular envelope }\end{array}$ \\
\hline 261 & Alba & Viséan & $\begin{array}{c}1.5(7) ; 2(3) \\
2.5(1)\end{array}$ & 1.7 & 0.34 & $<50-65$ & sugary, grey patina & - \\
\hline 262 & Alba & Viséan & $1.5(3) ; 2(3)$ & 1.8 & 0.27 & $50-70$ & sugary, grey patina & - \\
\hline 263 & $\begin{array}{l}\text { Cuera Limestones } \\
\text { (lower) }\end{array}$ & Bashkirian & $1.5(8) ; 2(7)$ & 1.7 & 0.26 & $<50-65$ & sugary, grey patina & \\
\hline
\end{tabular}

Table 2. Kübler index (KI) values from samples collected in the Ponga-Cuera unit

\begin{tabular}{|c|c|c|c|}
\hline $\begin{array}{l}\text { Locality } \\
\text { number }\end{array}$ & Formation & Age & $\mathrm{KI}(\Delta 2 \theta)$ \\
\hline 13 & Oville & $\begin{array}{l}\text { Upper Cambrian- } \\
\text { Lower Ordovician }\end{array}$ & 0.72 \\
\hline 14 & Oville & $\begin{array}{l}\text { Upper Cambrian- } \\
\text { Lower Ordovician }\end{array}$ & 0.96 \\
\hline 15 & Beleño & Lower Moscovian & 0.58 \\
\hline 16 & $\begin{array}{l}\text { Cuera Limestones } \\
\text { (lower) }\end{array}$ & Bashkirian & 0.72 \\
\hline 17 & Beleño & Lower Moscovian & 0.58 \\
\hline 18 & Beleño & Lower Moscovian & 0.58 \\
\hline 19 & Beleño & Lower Moscovian & 0.78 \\
\hline 20 & Beleño & Lower Moscovian & 0.66 \\
\hline 21 & Beleño & Lower Moscovian & 0.62 \\
\hline 41 & Beleño & Lower Moscovian & 1.01 \\
\hline 42 & Beleño & Lower Moscovian & 0.92 \\
\hline 43 & Beleño & Lower Moscovian & 1.14 \\
\hline 44 & Alba & Viséan & 0.79 \\
\hline $44 \mathrm{~A}$ & Barrios & Lower Ordovician & 0.76 \\
\hline 46 & Barrios & Lower Ordovician & 0.58 \\
\hline 50 & Barrios & Lower Ordovician & 1.01 \\
\hline
\end{tabular}

a value of 7 . In one case, the CAI shows a high dispersion, from 1 to 3.5 .

A sugary texture (dull to shiny, pitted or frosted surfaces) is the most common texture of the conodonts in the Ponga-Cuera unit. Less frequently, a smooth texture appears, usually displaying a shiny surface. When this texture is present, it always appears combined with sugary texture. It is also very common for the conodonts to appear bleached or/and smoked. A grey patina on the conodont surface is common. Basal cavities can be filled by apatite crystallites in many conodonts; sometimes they are associated with authigenic crystals of quartz.

When the sugary texture is observed under the scanning electron microscope, several microtextures can be distinguished that correspond to thin envelopes of apatite crystals that have grown over the conodont surface. Microtextures of external casts, of small crystals, and of irregular envelopes are the most common; they usually appear combined in the same conodont (Fig. 4). The microtexture of external casts consists of an irregular net of polygons developed on the surface of the conodonts, and whose sides are defined by apatite walls in which individual crystals are difficult to distinguish (Fig. 4a1, a2). The polygonal areas sometimes show the unaltered original microtexture, which can be composed of smooth or micro-ornamented surfaces. The microtexture of small crystals is defined by a thin cover of apatite 


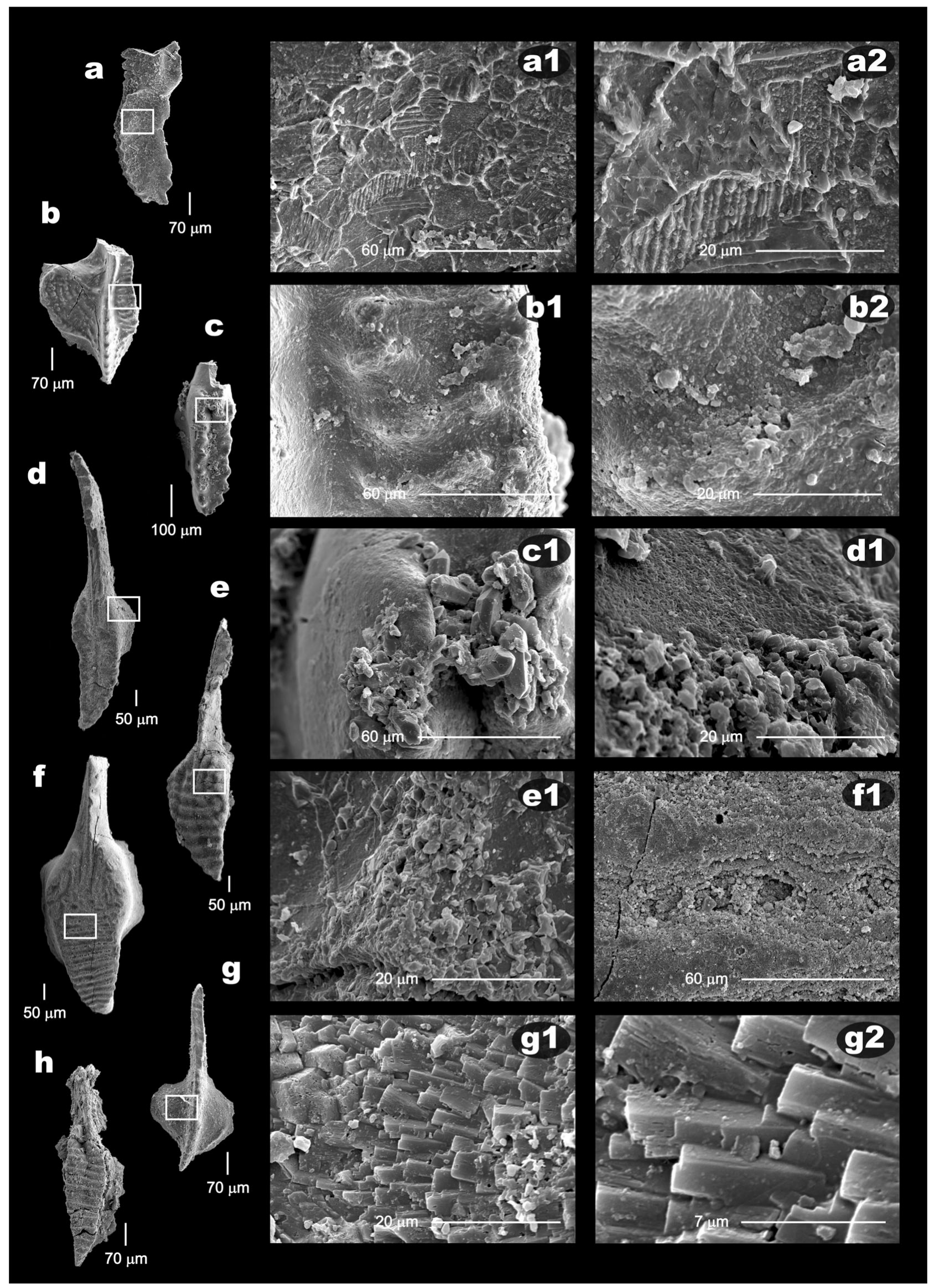

Figure 4. For caption see facing page. 
anhedral or subhedral crystals; it appears as much on the surfaces of the polygonal areas as on surfaces without polygons (Fig. 4b1, b2). The irregular envelope microtexture is formed by a heterogeneous partial or total cover of apatite and other minerals (mainly idiomorphic quartz and carbonates; Fig. 4c1, d1, e1, h). It also includes attached grains undissolved in the processing of the samples. A microtexture of large crystals (mainly formed by euhedral crystals developed on the oral surface of conodont; Fig. $4 \mathrm{~g} 1$, g2) and local dissolution microtextures (Fig. 4f1) occur less frequently. Microtextures of small and large crystals are in part coeval with the microtexture of external casts. The irregular envelope and dissolution microtextures are developed on the other microtextures. Conodonts from some grainstone rocks show irregular fractures without a specific pattern of distribution. These fractures cut the external casts, small crystals and large crystals of the corresponding microtextures. The parts of these broken conodonts are connected by an irregular envelope microtexture. A clear relation between texture or microtexture types and CAI values of the conodonts is not observed.

In agreement with the CAI data, the KI values measured on Cambrian-Ordovician and Carboniferous shales range between 0.58 and 1.14, indicating diagenetic conditions in all cases (Table 2). Mineralogical analyses of the $<2 \mu \mathrm{m}$ fraction of the pelitic rocks show that the major component is illite. There are also small amounts of chlorite, kaolinite and mixed layered illite/smectite and chlorite/vermiculite.

\section{Interpretation}

Except for very local anomalies, the Ponga-Cuera unit is characterized by a great uniformity in the CAI values, which indicates almost no thermal variation in the horizontal directions (Fig. 3). Hence, the thermal conditions of the rocks indicated by the CAI values are independent of the stratigraphic position of the samples. The most abundant CAI value is 1.5 , which requires a temperature between 50 and $90{ }^{\circ} \mathrm{C}$ (Epstein, Epstein \& Harris, 1977), depending on the heating time of the conodonts. Local CAI values close to 2 correspond to a temperature interval of $60-140{ }^{\circ} \mathrm{C}$ (after calibration: Epstein, Epstein \& Harris, 1977). The highest temperatures of these intervals require short periods of heating. In the present case, in which most of the CAI values are $<2$, the rocks were probably subject to the effects of relatively low temperatures (between 50 and $80^{\circ} \mathrm{C}$ ) for a long period, specifically from the Pennsylvanian (the burial age of the rocks) until the Cenozoic (the age of the final rock exhumation). Temperatures within this interval are sufficient to explain the most common CAI values $(\mathrm{CAI}<2)$ in the present case. Assuming a normal geothermal gradient (about $30^{\circ} \mathrm{C} / \mathrm{km}$ ), the low temperatures cited above would require a rock overburden no greater than $3 \mathrm{~km}$ in the area studied, which is a reasonable value for the stratigraphic thickness cited above. This overburden was due partially to burial and partially to tectonic superimposition. At the end of Moscovian times, the thickness of the Carboniferous succession was at least of $2300 \mathrm{~m}$, generating the first overburden by burial. The overburden increased during the first episodes of the Variscan thrusting, which took place during Moscovian-Gzhelian times. However, the thrusts of the area did not give rise to a discontinuity in the CAI values; this indicates limited displacement and tectonic superimposition or a subsequent heating event. As the Variscan deformation progressed, the erosion of the belt increased and tended to balance the overburden produced by the tectonic superimposition, resulting in the main Variscan exhumation of the unit taking place in Gzhelian times. During Permian times, the compressive tectonic regime of the orogen evolved to an extensional regime that was maintained until the beginning of the Alpine deformation. After the post-Variscan exhumation, burial associated with the cover (1300-2000 $\mathrm{m}$ thick) will have maintained the temperature of Carboniferous rocks above $50{ }^{\circ} \mathrm{C}$, and probably tended to homogenize the CAI values of the unit by increasing the lower values. Alpine fault displacement and tectonic superimposition are minor and have only local effects.

The near lack of horizontal thermal variation in the Ponga-Cuera unit extends southwards into a large part of the adjacent Picos de Europa unit and contrasts with the high CAI gradient observed in the southern part of this unit (Fig. 1). Here the temperature increases southwards and the ancaizone-diacaizone boundary is

Figure 4. Conodont microtextures of P1 elements in oral and lateral views from the Ponga-Cuera unit. (a) Idiognathoides sinuatus Harris \& Hollingsworth, CAI = 1.5 (sample 135, Barcaliente Fm.). (a1, a2) Microtexture of external casts showing casts of calcite twins in several polygons. (b) Gnathodus truyolsi Sanz-López et al., CAI $=1$ (sample 50, Barcaliente Fm.). (b1, b2) Microtexture of small crystals; several larger crystals above correspond to the microtexture of irregular envelope. (c) Declinognathodus japonicus (Igo \& Koike), CAI = 2 (sample 49, Barcaliente Fm.). (c1) Partial filling by microtexture of irregular envelope with bipiramidal authigenic quartz crystals. (d) Declinognathodus sp., CAI $=2$ (sample 63, Cuera Limestones). (d1) Isolated large anhedral crystals of the microtexture of irregular envelope (below) are over the microtexture of small crystals (above). (e) Idiognathodus sp., CAI = 2 (sample 155, Cuera Limestones). (e1) Fracture where crystals of the microtexture of irregular envelope grew above the margins. (f) Idiognathodus sp., CAI $=2$ (sample 238, Cuera Limestones). (f1) Detail of one dissolved ridge of the conodont ornamentation showing the internal lamellae of apatite crystals. (g) Lochriea ziegleri Nemirovskaya, Perret \& Meischner, CAI $=1$ (sample 53, Alba Fm.). (g1, g2) Somewhat oriented euhedral crystals forming the microtexture of large crystals. (h) Idiognathoides sinuatus Harris \& Hollingsworth, CAI = 1.5-2 (sample 155, Cuera Limestones); conodont showing fracturing and microtexture of irregular envelope. 
generally located immediately above the basal thrust of the unit (Bastida et al. 2004; García-López et al. 2007). This gradient is independent of the stratigraphic order, and this suggests a thermal source located to the south of the Picos de Europa unit, that is to say, in the Pisuerga-Carrión unit (Fig. 1), where post-Variscan igneous activity with development of granitoids exists. The influence of this thermal event did not reach the area studied in this paper.

Results from CAI values of the Ponga-Cuera unit agree not only with those obtained from the KI values, but also with the coal rank (bituminous $\mathrm{C}$ coal) and vitrinite reflectance $(\operatorname{Rr} \%$ between 0.8 and 0.9$)$ values obtained from the unconformable Kasimovian rocks located just to the south of the study area (Colmenero et al. 2008). In agreement with the correlations between the thermal indicators (e.g. Teichmüller, 1987; Merriman \& Frey, 1999; García-López et al. 2001), these indicate diagenetic (or diacaizonal) conditions for the Ponga-Cuera unit.

Post-Variscan hydrothermal activity developed mainly during Permian times (Martínez García et al. 1991; Paniagua et al. 1993; Weh et al. 2001; Bastida et al. 2004; García-López et al. 2007) and gave rise to ore deposits and to dolomitization and silicification in the Ponga-Cuera and Picos de Europa units. The pressures estimated from the analysis of the ore deposits (20-60 MPa; Luque \& Martínez García, 1985; Luque, Martínez-García \& Ruiz, 1990) agree with the thickness of the overburden $(1-3 \mathrm{~km})$ required to reach the temperature that gave rise to the CAI values obtained in the area with a normal geothermal gradient. The activity involved low-temperature fluid flow (100 $200{ }^{\circ} \mathrm{C}$ in the study area) along fractures, and had little influence in the general thermal regime of the PongaCuera unit, in the CAI and the KI values. Hence, the effects of this hydrothermal activity can only be observed in certain localities close to faults, joint sets or sedimentary boundaries between different lithologies. In these cases, the CAI values are $\geq 2$ or have high CAI dispersion, sometimes with two apparent modal CAI values. The high mode (CAI 7) is due to the strong corrosion of the conodonts (which is well observed under the scanning electron microscope) with loss of organic and mineral matter. The lower mode may be the result of colour alteration retarded by pressurized fluids of hydrothermal origin (Rejebian, Harris \& Huebner, 1987). The small difference in temperature between the mineralizing hydrothermal fluids and the host rock, the small size of the mineralized bodies, and probably the short duration of the hydrothermal processes, are the main reasons for the lack of appreciable thermal anomalies around the ore deposits (Fig. 3).

The Ponga-Cuera unit has a low-thermal history and widespread development of dissolution and neocrystallization conodont microtextures. In other areas these alterations have usually been related to dolomitization, deformation and/or hydrothermal fluids, with CAI $\geq 3$ for the two latter processes (Rejebian, Harris \& Huebner, 1987; Fuchs, 1989; Helsen, 1995, 1997;
Janssen, Friedel \& Paech, 1988; Königshof, 1992, 2003; Nöth, 1998; Königshof \& Boncheva, 2005). In the present case, dolomitization and deformation can be rejected because the host rocks of all the conodont samples have always been unstrained limestones with low CAI values. In addition, hydrothermal activity had only local influence in the development of the colour and microtextures of the conodonts. The studied microtextures were widely developed in diagenetic conditions, and they are difficult to explain as a result of fluids circulating through a net of fissures inside a lithified rock. An extended fluid mobility for the widespread development of microtextures could take place before the complete lithification of the sediment. In this sense, broken conodonts with irregular fracturing from some beds suggest a pre-lithification compaction process that affected the microtextures cut by the fractures (external casts, small crystal and large crystal microtextures). Features observed on the microtexture of external casts agree with its early development. The walls that define the microtexture of external casts are due to apatite neocrystallization on the conodont surface next to the spaces between adjoining grains (mainly calcite crystals) in the host rock. This process would be favoured by the porosity typical of an unconsolidated sediment. Helsen (1995) offered an explanation comparable to that given above, whereas other authors attributed this structure to pressure solution (e.g. Burnett, 1988). In the present study, there are no microtextural traces of this mechanism. In some cases, the polygonal areas show the typical appearance of the unaltered surfaces of the conodont, and in other cases, they show the development of a microtexture of small crystals with no dissolution features present in these areas. Occasionally, straight rows of small apatite crystals appear in the microtexture of external casts. These are probably the casts of calcite twins of grains adjacent to the conodont.

The microtexture of large crystals has a local distribution (Fig. 3) and is associated with conodonts coming from only a few formations (common in Alba Formation, rare in the Cuera Limestones and Barcaliente Formation). These are formations with carbonate beds (packstones or grainstones) enriched with iron oxide-hydroxide grains, and with sedimentary pore spaces where crystallization of large idiomorphic apatite crystals is easier.

All of the microtextures described above involve apatite neocrystallization, so it would be interesting to know which phosphate source made the precipitation of this mineral possible. Recent phosphate concentrations in marine pore-water may be due to release of the organic matter by microbial degradation, desorption from iron oxides, and fish debris dissolution (Suess, 1981; Froelich et al. 1988; Babu \& Nath, 2005). In the present case, it is possible that part of the phosphate also came from the more soluble parts of conodonts, as has been described for other localities of the Cantabrian zone (Sanz-López, Blanco-Ferrera \& García-López, 2009). 
Microtextures in conodonts with low CAI values from the Ponga-Cuera unit indicate the reactivity of apatite under early diagenesis. This agrees with the diagenetic modifications in the geochemical/isotopic composition of conodonts with CAI values $<2$ described by Holmden et al. (1996), Ebneth et al. (1997) and John, Cliff \& Wignall (2008).

\section{Conclusions}

CAI values obtained in the Ponga-Cuera unit display great uniformity and are mainly $<2$. This is indicative of diacaizonal conditions, and agrees with the diagenetic conditions inferred from KI data obtained from several samples of shales, and with the coal rank and the vitrinite reflectance data from rocks located just to the south of the area studied in this paper. These conditions require an overburden $<3 \mathrm{~km}$, and were probably maintained from the Late Carboniferous (burial age of rocks) until the Cenozoic (age of final rock exhumation). To reach the CAI of the studied area, this long period of heating would involve low temperatures $\left(<100^{\circ} \mathrm{C}\right)$.

The most common microtextures of the conodonts studied are external casts and small apatite crystals. Other textures present are the irregular envelope, large crystal and dissolution microtextures. These microtextures developed independently of the CAI values. The external cast and small and large apatite crystal microtextures were mainly the result of neocrystallization that took place before the complete lithification of the carbonate sediment. Irregular envelope and dissolution microtextures developed later.

Post-Variscan epithermal activity mainly occurred during Permian times and only produced small alterations in the general thermal regime of the unit. None the less, local CAI anomalies involving higher values (2 to 3), high CAI dispersion in the same sample, and dissolution microtextures can all be related to this activity, mainly when they are located near faults or lithological discontinuities. Whatever the case, the CAI anomalies are not completely coincident with the location of ore deposits in the area.

Acknowledgements. The work has been supported by Spanish projects CGL2006-06401 and CGL-2009-09109, funded by the 'Ministerio de Educación y Ciencia', 'Ministerio de Ciencia e Innovación' and 'Fondo Europeo de Desarrollo Regional' (FEDER). We are grateful to P. Königshof and an anonymous reviewer for valuable suggestions that greatly improved the original manuscript. We also thank to A. Castro and C. Sueiro (University of A Coruña) for their assistance with the scanning electron microscope, and Robin Walker for his language revision.

\section{References}

BABU, C. P. \& NATH, B. N. 2005. Processes controlling forms of phosphorous in surficial sediments from the eastern Arabian Sea impinged by varying bottom water oxygenation conditions. Deep-Sea Research II 52, 1965-80.

Bastida, F., Blanco-Ferrera, S., García-López, S., SANZ-LÓPEZ, J. \& VALÍN, M. L. 2004. Transition from diagenesis to metamorphism in a calcareous tectonic unit of the Iberian Variscan belt (Central massif of the Picos de Europa, NW Spain). Geological Magazine 141, 617-28.

BAstida, F., BRIME, C., GARcía-LóPez, S. \& SARMIENTO, G. N. 1999. Tectono-thermal evolution in a region with thin skinned tectonics: the western nappes in the Cantabrian Zone (Variscan belt of NW Spain). International Journal of Earth Sciences 88, 38-48.

BuRnetT, R. D. 1988. Physical and chemical changes in conodonts from contact-metamorphosed limestones. Irish Journal of Earth Sciences 9, 79-119.

COLMENERO, J. R., SUÁREZ-RuIZ, I., FERNÁNDEZ-SuÁrEZ, J., BARBA, P. \& Llorens, T. 2008. Genesis and rank distribution of Upper Carboniferous coal basins in the Cantabrian Mountains, Northern Spain. International Journal of Coal Geology 76, 187204.

Ebneth, S., Diener, A., Buhl, D. \& Veizer, J. 1997. Strontium isoptope systematics of conodonts: Middle Devonian, Eifel Mountains, Germany. Palaeogeography, Palaeoclimatology, Palaeoecology 132, 7996.

Epstein, A. G., Epstein, J. B. \& Harris, L. D. 1977. Conodont Colour Alteration - an index to organic metamorphism. U.S. Geological Survey Professional Paper 995, 1-27.

Froelich, P. N., Arthur, M. A., Burnett, W. C., Deakin, M., Hensley, V., JahnKe, R., KaUl, L., KIM, K.-H., RoE, H., Soutar, A. \& VATHAKANON, C. 1988. Early diagenesis of organic matter in Peru continental margin sediments: phosphorite precipitation. Marine Geology 80, 309-43.

FuCHS, A. 1989. Zur Bestimmung der Versenkungstemperatur im Ordovizium Thüringens und Skandinavens mit Hilfe der Conodontenfarbe. Neues Jahrbuch Geologie und Paläontologie Monatshefte 1989-7, 390-9.

García-LÓPez, S., BASTidA, F., Aller, J. A. \& SANZLÓPEZ, J. 2001. Geothermal paleogradients and metamorphic zonation from the conodont colour alteration index (CAI). Terra Nova 13, 79-83.

García-LóPez, S., Bastida, F., Brime, C., Aller, J., VAlín, M. L., SANZ-LóPez, J., MÉndez, C. A. \& MEnÉndez-Álvarez, J. R. 1999. Los episodios metamórficos de la Zona Cantábrica y su contexto estructural. Trabajos de Geología de la Universidad de Oviedo 21, 177-87.

GARCÍA-LÓPEZ, S., Brime, C., BASTIDA, F. \& SARMIENTO, G. N. 1997. Simultaneous use of thermal indicators to analyse the transition from diagenesis to metamorphism: an example from the Variscan Belt of northwest Spain. Geological Magazine 134, 32334.

GARCÍA-LÓPEZ, S., BRIME, C., VALÍN, M. L., SANZLÓPEZ, J., BASTIDA, F., Aller, J. \& BlANCO-FERRERA, S. 2007. Tectonothermal evolution of a foreland fold and thrust belt: the Cantabrian Zone (Iberian Variscan belt, NW Spain). Terra Nova 19, 46975.

Guggenheim, S., Bain, D. C., Bergaya, F., Brigatti, M. F., Drits, V. A., Eberlt, D. D., Formoso, M. M. L., Galán, E., Merriman, R. J., Peacor, D. R., StanjeK, H. \& Watanabe, T. 2002. Report of the Association Internationale pour l'Étude des Argiles 
(AIPEA) Nomenclature Committee for 2001: order, disorder, and crystallinity in phyllosilicates and the use of the "Crystallinity Index". Clays and Clay Minerals 50, 406-9.

GutiérRez-Claverol, M. \& Luque, C. 2000. La minería en los Picos de Europa. Oviedo: Noega, $303 \mathrm{pp}$.

Helsen, S. 1995. Conodont alteration in DevonoCarboniferous dolomites from southern Belgium. Geologie in Mijnbouw 74, 225-34.

HELSEN, S. 1997. Conodont colour alteration in Walsortian buildups of the type area (Lower Carboniferous, Belgium). Annales de la Société géologique de Belgique 120, 155-82.

Holmden, C., Creaser, R. A., Muehlenbachs, K., Bergstrom, S. M. \& Leslie, S. A. 1996. Isotopic and elemental systematics of $\mathrm{Sr}$ and $\mathrm{Nd}$ in 454 Ma biogenic apatites: implications for paleoseawater studies. Earth and Planetary Science Letters 142, 425 37.

Janssen, Ch., Friedel, C.-H. \& PaeCh, H.-J. 1988. Zur Schieferung in devonischen Riffkarbonaten. Zeitschrift für Geologische Wissenschaften 16, 721-37.

JEPPSSON, L. \& ANEHUS, R. 1995. A buffered formic acid technique for conodont extraction. Journal of Paleontology 69, 790-4.

John, E. H., ClifF, R. \& Wignall, P. B. 2008. A positive trend in seawater ${ }^{87} \mathrm{Sr} /{ }^{86} \mathrm{Sr}$ values over the Early-Middle Frasnian boundary (Late Devonian) recorded in wellpreserved elements from the Holy Cross Mountains, Poland. Palaeogeography, Palaeoclimatology, Palaeoecology 269, 166-75.

JULIVERT, M. 1971. Décollements tectonics in the Hercynian Cordillera of NW Spain. American Journal of Science 270, 1-29.

KISCH, H. J. 1991. Illite crystallinity: recommendations on sample preparation, X-ray diffraction settings and interlaboratory settings. Journal of Metamorphic Geology $\mathbf{9}$, 665-70.

KÖNIGSHOF, P. 1992. Der Farbänderungsindex von Conodonten (CAI) in paläozoichen Gesteinen (Mitteldevon bis Unterkarbon) des Rheinischen Schiefergebirges - eine Ergänzung zur Vitriniteflexion. Courier Forschungsinstitut Senckenberg 146, 1-115.

KÖNIGSHOF, P. 2003. Conodont deformation patterns and textural alteration in Paleozoic conodonts: examples from Germany and France. Senckenbergiana lethaea $\mathbf{8 3}$, 149-56.

KÖNIGSHOF, P. \& BONCHEVA, I. 2005. Maturation patterns in Palaeozoic rocks of north-eastern Bulgaria based on conodont colour alteration index (CAI) data. Bulletin of Geosciences 81, 223-37.

LOREDO, J. \& GARCÍA IGLESIAS, J. 1981. Análisis microtermométrico del yacimiento de fluorita de "Pie de Potro" (Caravia, Asturias). Revista de Minas 2, 117 25.

LUQUe, C. \& GUTIÉRREZ ClaVEROL, M. 2006. La minería del mercurio en Asturias. Oviedo: Carlos Luque \& Manuel Gutiérrez Claverol, $554 \mathrm{pp}$.

LuQue, C. \& MARTíneZ-GARCíA, E. 1985. Análisis tectometalogenético de las mineralizaciones en el Carbonífero de la Zona Cantábrica (NW de España). $C$. R. X Congreso Internacional de Geología y Estratigrafia del Carbonifero, Madrid (1983), 95-104.

LuQue, C., Martínez-García, E. \& Ruiz, F. 1990. Metallogenesis. In Pre-Mesozoic Geology of Iberia (eds R. D. Dallmeyer \& E. Martínez-García), pp. 80-7. Berlin: Springer-Verlag.
MARQUínEZ, J. 1989. Mapa geológico de la región del Cuera y los Picos de Europa. Trabajos de Geología de la Universidad de Oviedo 18, 137-44.

Martín-IZARD, A., PAniaguA, A. \& ARIAS, D. 1995. Yacimientos minerales: modelos de formación. In Geología de Asturias (eds C. Aramburu \& F. Bastida), pp. 139-52. Oviedo: Trea.

MARTíNEZ-GARCÍA, E. 1981. Tectónica y mineralizaciones pérmicas en la Cordillera Cantábrica Oriental (Noroeste de España). Cuadernos do Laboratorio Xeolóxico de Laxe 2, 263-71.

MARTíNEZ-GARCÍA, E. 1983. Permian mineralization in the Cantabrian Mountains (North-West Spain). In Mineral deposits of the Alps and of the Alpine epoch in Europe (ed. H.-J. Schneider), pp. 259-74. Berlin-Heidelberg: Springer-Verlag.

MARTíneZ-GARCíA, E. \& TEJerinA, L. 1985. Fluorspar deposits associated with Carboniferous and Permian rocks in Asturias and León (Northwest Spain). C. R. IX International Congress of Stratigraphy and Geology of the Carboniferous, Washington-Urbana/Champaign $5,467-78$.

Martínez-García, E., Luque, C., Burkhardt, R. \& GutiérRez-Claverol, M. 1991. Hozarco: un ejemplo de mineralización de $\mathrm{Pb}-\mathrm{Zn}-\mathrm{Hg}$ de edad pérmica (Cordillera Cantábrica, NW de España). Boletín de la Sociedad Española de Mineralogía 14, 107-16.

MartínEZ-García, E., ANTONA, J. F., GARCÍA SÁNCHEZ, A. \& QUIROGA DE LA VEGA, J. L. 2004. Tectonic and metallogenetic significance of sedimentary manganese deposits in the Eastern Cantabrian domain, Asturias, Northwestern Spain. International Geology Review 46, 273-83.

MERriman, R. J. \& FREY, M. 1999. Patterns of very lowgrade metamorphism in metapelitic rocks. In Low grade metamorphism (eds M. Frey \& D. Robinson), pp. 61107. Oxford: Blackwell Science.

Navarro, D., Leyva, F., Villa, E. \& Granados, L. 1986. Mapa Geológico de España a escala 1:50000 Ribadesella (31, 15-4). Segunda serie, Primera edición. Madrid: ITGE, 109 pp.

NÖTH, S. 1998. Conodont color (CAI) versus microcrystalline and textural changes in Upper Triassic conodonts from Northwest Germany. Facies 38, 165-74.

Paniagua, A., Fontboté, L., Fenoll, P., Fallick, A. E., Moreiras, D. B. \& Corretgé, L. G. 1993. Tectonic setting, mineralogical characteristics and age dating of a new type of epithermal carbonate-hosted, precious metal-five element deposits: the Villamanín Area (Cantabrian Zone, Northern Spain). In Current Research in Geology Applied to Ore Deposits (eds P. Fenoll Hach-Ali, J. Torres-Ruiz \& F. Gervilla), pp. 5314. Granada: Departamento de Mineralogía y Petrología.

Rejebian, V. A., HARris, A. G. \& Huebner, J. S. 1987. Conodont colour and textural alteration: an index to regional metamorphism and hydrothermal alteration. Geological Society of America Bulletin 99, 471-9.

SÁNCHEZ DE PosadA, L. C., MARTínez Chacón, M. L., Méndez, C. A., Menéndez-Álvarez, J. R., Río, M., Rodríguez, S., Truyols, J. \& Villa, E. 1996. El Carbonífero marino del ámbito astur-leonés (Zona Cantábrica): Síntesis paleontológica. Revista Española de Paleontología Número Extraordinario, 82-96.

SANZ-LóPEZ, J., BlANCO-FERRERA, S. \& GARCÍALÓPEZ, S. 2009. Changes of the apatite overgrowth on the surface of diacaizonal conodonts from the Cantabrian Zone (Spain). International Conodonts 
Symposium (ICOS 2009) Abstracts. Permophiles 53, Supplement 1, 46.

SUESS, E. 1981. Phosphate regeneration from sediments of the Peru continental margin by dissolution of fish debris. Geochimica et Cosmochimica Acta 45, 577-88.

TEICHMÜLlER, M. 1987. Organic material and very lowgrade metamorphism. In Low temperature metamorphism (ed. M. Frey), pp. 114-61. New York: Chapman and Hall.
TosAL, J. M. 1968. Relaciones zócalo-cobertera en el límite de las provincias de Oviedo y Santander. Breviora Geológica Artúrica 12, 9-14.

Weh, A., Krumm, S., Clauer, N. \& Keller, M. 2001. The late orogenic history of the southeastern Cantabrian Mountains: illite-crystallinity and $\mathrm{K}-\mathrm{Ar}$ data. Meeting of the European Union of Geosciences XI (EUG XI), Strasbourg. Journal of Conference Abstracts 6, 233. 